\title{
Correction to: Transfer Learning for Semi-supervised Classification of Non-stationary Data Streams
}

\author{
Yimin Wen, Qi Zhou, Yun Xue, and Chao Feng
}

Correction to:

Chapter "Transfer Learning for Semi-supervised

Classification of Non-stationary Data Streams"

in: H. Yang et al. (Eds.): Neural Information Processing, CCIS 1333, https://doi.org/10.1007/978-3-030-63823-8_54

The originally published version of the chapter 54 consisted typesetting errors in Tables 2-5. This has been corrected. 